\title{
Oscillation of higher-order differential equations with distributed delay
}

\section{O. Bazighifan ${ }^{1 *}$ D, E.M. Elabbasy ${ }^{2}$ and O. Moaaz ${ }^{2}$}

${ }^{\text {*Correspondence: }}$
o.bazighifan@gmail.com
${ }^{1}$ Department of Mathematics,
Faculty of Education, Hadhramout
University, Hadhramout, Yemen
Full list of author information is
available at the end of the article

available at the end of the article

\section{Abstract \\ This paper deals with the oscillation properties of higher-order nonlinear differential equations with distributed delay}

$$
\left[b(\ell)\left(y^{(n-1)}(\ell)\right)^{\gamma}\right]^{\prime}+\int_{c}^{d} q(\ell, \xi) y^{\gamma}(g(\ell, \xi)) d(\xi)=0, \quad \ell \geq \ell_{0}
$$

under the condition

$$
\int_{\ell_{0}}^{\infty} \frac{1}{b^{\frac{1}{\gamma}}(\ell)} d \ell<\infty
$$

We obtain new oscillation criteria by employing a refinement of the generalized Riccati transformations and new comparison principles. We provide some examples to illustrate the main results.

MSC: $34 \mathrm{~K} 10 ; 34 \mathrm{~K} 11$

Keywords: Higher-order; Distributed delay differential equations; Oscillatory solutions; Nonoscillatory solutions

\section{Introduction}

In this work, we investigate the oscillation behavior of solutions to the higher-order nonlinear differential equation with distributed delay of the form

$$
\left[b(\ell)\left(y^{(n-1)}(\ell)\right)^{\gamma}\right]^{\prime}+\int_{c}^{d} q(\ell, \xi) y^{\gamma}(g(\ell, \xi)) d(\xi)=0, \quad \ell \geq \ell_{0} .
$$

We assume that the following assumptions hold:

$\left(A_{1}\right) b \in C^{1}\left[\ell_{0}, \infty\right), b^{\prime}(\ell) \geq 0, b(\ell)>0, \gamma$ is a quotient of odd positive integers;

$\left(A_{2}\right) q(\ell, \xi), q(\ell, \xi) \in C\left(\left[\ell_{0}, \infty\right) \times[c, d], \mathbb{R}\right), q(\ell, \xi)$ is positive, $g(\ell, \xi)$ is a nondecreasing function in $\xi, g(\ell, \xi) \leq \ell$, and $\lim _{\ell \rightarrow \infty} g(\ell, \xi)=\infty$.

By a solution of equation (1.1) we mean a function $y \in C^{n-1}\left[\ell_{y}, \infty\right), L_{y} \geq \ell_{0}$, that has the property $b(\ell)\left(y^{n-1}(\ell)\right)^{\gamma} \in C^{1}\left[L_{y}, \infty\right)$ and satisfies equation (1.1) on $\left[L_{y}, \infty\right)$. We consider only solutions $y$ of equation (1.1) that satisfy $\sup \{|y(\ell)|: \ell \geq L\}>0$ for all $\ell>L_{y}$. We assume that (1.1) possesses such a solution. A solution of (1.1) is called oscillatory if it has

(c) The Author(s) 2019. This article is distributed under the terms of the Creative Commons Attribution 4.0 International License (http://creativecommons.org/licenses/by/4.0/), which permits unrestricted use, distribution, and reproduction in any medium, provided you give appropriate credit to the original author(s) and the source, provide a link to the Creative Commons license, and indicate if changes were made. 
arbitrarily large zeros on $\left[L_{y}, \infty\right)$, and otherwise it is called nonoscillatory. Equation (1.1) is said to be oscillatory if all its solutions are oscillatory.

The problem of the oscillation of fourth- and higher-order differential equations have been widely studied by many authors, who have provided many techniques for obtaining oscillatory criteria for fourth- and higher-order differential equations. We refer the reader to the related books $[1,4,9-11,19]$ and to the papers [2, 3, 5, 8, 12-24]. In the follows, we present some related results that served as a motivation for the contents of this paper.

Zhang et al. [22] studied the oscillation behavior of the higher-order nonlinear differential equation

$$
\left[b(\ell)\left(y^{(n-1)}(\ell)\right)^{\gamma}\right]^{\prime}+q(\ell) y^{\beta}(\tau(\ell))=0 .
$$

Tunc and Bazighifan [21] studied the oscillatory behavior of the fourth-order nonlinear differential equation with a continuously distributed delay

$$
\left[b(\ell)\left(y^{\prime \prime \prime}(\ell)\right)^{\beta}\right]^{\prime}+\int_{c}^{d} q(\ell, \tau) x^{\beta}(g(\ell, \tau)) d(\tau)=0
$$

Bazighifan [4] considered the oscillatory properties of the higher-order differential equation

$$
\left[b(\ell)\left(y^{(n-1)}(\ell)\right)^{\gamma}\right]^{\prime}+q(\ell) f(y(\tau(\ell)))=0
$$

under the conditions

$$
\int_{\ell_{0}}^{\infty} \frac{1}{b^{\frac{1}{\gamma}}(\ell)} d \ell=\infty
$$

and

$$
\int_{\ell_{0}}^{\infty} \frac{1}{b^{\frac{1}{\gamma}}(\ell)} d \ell<\infty
$$

Moaaz et al. [19] considered the fourth-order differential equations of the form

$$
\left[b(\ell)\left(y^{\prime \prime \prime}(\ell)\right)^{\alpha}\right]^{\prime}+\int_{a}^{b} q(\ell, \xi) f(x(g(\ell, \xi))) d(\xi)=0
$$

Elabbasy et al. [6, 7] and Zhang et al. [24] examined the oscillation of the fourth-order nonlinear delay differential equation

$$
\left[b(\ell)\left(y^{\prime \prime \prime}(\ell)\right)^{\alpha}\right]^{\prime}+q(\ell) y^{\alpha}(\ell)=0 .
$$

Our aim in the present paper is to employ the Riccatti technique to establish some new conditions for the oscillation of all solutions of equation (1.1) under condition (1.2). We present some examples to illustrate our main results.

The proof of our main results are essentially based on the following lemmas. 
Lemma 1.1 (See [1], Lemma 2.2.3) Let $z \in\left(C^{n}\left[\ell_{0}, \infty\right], \mathbb{R}^{+}\right)$and assume that $z^{(n)}$ is of fixed sign and not identically zero on a subray $\left[\ell_{0}, \infty\right]$. If, moreover, $z(\ell)>0, z^{(n-1)}(\ell) z^{(n)}(\ell) \leq 0$, and $\lim _{\ell \rightarrow \infty} z(\ell) \neq 0$, then, for every $\lambda \in(0,1)$, there exists $\ell_{\lambda} \geq \ell_{0}$ such that

$$
z(\ell) \geq \frac{\lambda}{(n-1)} \ell^{n-1}\left|z^{(n-1)}(\ell)\right| \quad \text { for } \ell \in\left[\ell_{\lambda}, \infty\right) .
$$

Lemma 1.2 (See [19], Lemma 1.1) If the function $z$ satisfies $z^{(i)}>0, i=0,1, \ldots, n$, and $z^{(n+1)}<0$, then

$$
\frac{z(\ell)}{\ell^{n} / n !} \geq \frac{z^{\prime}(\ell)}{\ell^{n-1} /(n-1) !} .
$$

Lemma 1.3 (See [21], Lemma 1) Let $\beta \geq 1$ be a ratio of two numbers, and let $U$ and $V$ be constants. Then

$$
U y-V y^{\frac{\beta+1}{\beta}} \leq \frac{\beta^{\beta}}{(\beta+1)^{\beta+1}} \frac{U^{\beta+1}}{V^{\beta}}, \quad V>0 .
$$

\section{Main results}

In this section, we establish some oscillation criteria for equation (1.1). We are now ready to state and prove the main results. For convenience, we denote

$$
\begin{aligned}
& R(\ell)=\int_{\ell_{0}}^{\infty} \frac{1}{b^{1 / \gamma}(s)} d s, \\
& \delta_{+}^{\prime}(\ell)=\max \left\{0, \delta^{\prime}(\ell)\right\}, \\
& Q(\ell)=\int_{c}^{d} q(\ell, \xi) d(\xi)
\end{aligned}
$$

and

$$
\sigma(v)=\int_{v}^{\infty} Q(s)(g(s, c) / s)^{3 \gamma} d v .
$$

Theorem 2.1 Let $\left(A_{1}\right),\left(A_{2}\right)$, and (1.2) hold. Assume that there exists a positive function $\delta \in C^{1}\left[\ell_{0}, \infty\right)$ such that

$$
\int_{\ell_{0}}^{\infty}\left[\delta(s) \frac{1}{(n-3) !} \int_{\ell}^{\infty}(v-\ell)^{(n-3)} \int_{v}^{\infty}\left[\frac{1}{b(v)} \sigma(s)\right]^{1 / \gamma} d v d v+\frac{\left(\left(\delta^{\prime}(s)\right)_{+}\right)^{2}}{4 \delta(s)}\right] d s=\infty .
$$

If

$$
\int_{\ell_{0}}^{\infty}\left[Q(s)\left(\frac{\lambda_{2}}{(n-2) !} g^{n-2}(s, c)\right)^{\gamma} R^{\gamma}(s)-\left(\frac{\gamma}{\gamma+1}\right)^{\gamma+1} \frac{b^{-1 / \gamma}(s)}{R(s)}\right] d s=\infty
$$

for some constant $\lambda_{2} \in(0,1)$, then every solution of $(1.1)$ is oscillatory.

Proof Assume that (1.1) has a nonoscillatory solution $y$. Without loss of generality, we can assume that $y(\ell)>0$. It follows from (1.1) that there exist tow possible cases for $\ell \geq \ell_{1}$, where $\ell_{1} \geq \ell_{0}$ is sufficiently large: 
Case 1: $y^{\prime}(\ell)>0, y^{(n-1)}(\ell)>0, y^{(n)}(\ell)<0,\left(b\left(y^{(n-1)}\right)^{\gamma}\right)^{\prime}(\ell) \leq 0$;

Case 2: $y^{\prime}(\ell)>0, y^{(n-2)}(\ell)>0, y^{(n-1)}(\ell)<0,\left(b\left(y^{(n-1)}\right)^{\gamma}\right)^{\prime}(\ell) \leq 0$.

Assume that case 1 holds. By Lemma 1.1 we find that $y(\ell) \geq(\ell / 3) y^{\prime}(\ell)$, and hence

$$
\frac{y(g(\ell, c))}{y(\ell)} \geq \frac{g^{3}(\ell, c)}{\ell^{3}}
$$

Integrating (1.1) from $\ell$ to $u$, we obtain

$$
\begin{aligned}
& b(u)\left(y^{(n-1)}(u)\right)^{\gamma}-b(\ell)\left(y^{(n-1)}(\ell)\right)^{\gamma} \\
&=-\int_{\ell}^{u} Q(s) y^{\gamma}(g(s, \xi)) d s \\
& \leq-y^{\gamma}(\ell) \int_{\ell}^{u} \int_{c}^{d} q(s, \xi)(g(s, \xi) / \ell)^{3 \gamma} d(\xi) d s .
\end{aligned}
$$

Letting $u \rightarrow \infty$, we see that

$$
b(\ell)\left(y^{(n-1)}(\ell)\right)^{\gamma} \geq y^{\gamma}(\ell) \sigma(\ell) .
$$

By virtue of $y^{\prime}(\ell)>0, g(\ell, \xi) \leq \ell$, and (2.3), we obtain

$$
y^{(n-1)}(\ell) \geq y(\ell)\left[\frac{1}{b(\ell)} \sigma(s)\right]^{1 / \gamma} .
$$

Integrating again from $\ell$ to $\infty$, we obtain

$$
y^{(n-2)}(\ell) \leq-y(\ell) \int_{\ell}^{\infty}\left[\frac{1}{b(\nu)} \sigma(s)\right]^{1 / \gamma} d \nu .
$$

Integrating $n-3$ times from $\ell$ to $\infty$, we find

$$
y^{\prime \prime}(\ell) \geq \frac{y(\ell)}{(n-3) !} \int_{\ell}^{\infty}(v-\ell)^{(n-3)} \int_{v}^{\infty}\left[\frac{1}{b(v)} \sigma(s)\right]^{1 / \gamma} d v d v .
$$

Define the function

$$
\omega(\ell):=\delta(\ell) \frac{y^{\prime}(\ell)}{y(\ell)} .
$$

Then $\omega(\ell)>0$ for $\ell \geq \ell_{1}$, and

$$
\omega^{\prime}(\ell):=\delta^{\prime}(\ell) \frac{y^{\prime}(\ell)}{y(\ell)}+\delta(\ell) \frac{y^{\prime \prime}(\ell) y(\ell)-\left(y^{\prime}(\ell)\right)^{2}}{y^{2}(\ell)} .
$$

From (2.5) and (2.6) it follows that

$$
\begin{aligned}
\omega^{\prime}(\ell) \leq & -\delta(\ell) \frac{1}{(n-3) !} \int_{\ell}^{\infty}(v-\ell)^{(n-3)} \int_{v}^{\infty}\left[\frac{1}{b(v)} \sigma(s)\right]^{1 / \gamma} d v d v \\
& +\frac{\left(\delta^{\prime}(\ell)\right)_{+}}{\delta(\ell)} \omega(\ell)-\frac{1}{\delta(\ell)} \omega^{2}(\ell) .
\end{aligned}
$$


Hence we have

$$
\begin{aligned}
\omega^{\prime}(\ell) \leq & -\delta(\ell) \frac{1}{(n-3) !} \int_{\ell}^{\infty}(v-\ell)^{(n-3)} \int_{v}^{\infty}\left[\frac{1}{b(v)} \sigma(s)\right]^{1 / \gamma} d v d v \\
& +\frac{\left(\left(\delta^{\prime}(\ell)\right)_{+}\right)^{2}}{4 \delta(\ell)} .
\end{aligned}
$$

Integrating (2.9) from $\ell_{1}$ to $\ell$, we get

$$
\int_{\ell_{1}}^{\ell}\left(\delta(s) \frac{1}{(n-3) !} \int_{\ell}^{\infty}(v-\ell)^{(n-3)} \int_{v}^{\infty}\left[\frac{1}{b(v)} \sigma(s)\right]^{1 / \gamma} d v d v+\frac{\left(\left(\delta^{\prime}(s)\right)_{+}\right)^{2}}{4 \delta(s)}\right) d s \leq \omega\left(\ell_{1}\right)
$$

for all large $\ell$, which contradicts (2.1).

Assume that case 2 holds. Noting that $b(\ell)\left(y^{(n-1)}(\ell)\right)^{\gamma}$ is nonincreasing, we have that

$$
b(s)\left(y^{(n-1)}(s)\right)^{\gamma} \leq b(\ell)\left(y^{(n-1)}(\ell)\right)^{\gamma}
$$

for all $s \geq \ell \geq \ell_{1}$. This yields

$$
y^{(n-1)}(s) \leq\left[b(\ell)\left(y^{(n-1)}(\ell)\right)^{\gamma}\right]^{1 / \gamma} \frac{1}{b^{1 / \gamma}(s)} .
$$

Integrating this inequality from $\ell$ to $u$, we get

$$
y^{(n-2)}(u)-y^{(n-2)}(\ell) \leq\left[b(\ell)\left(y^{(n-1)}(\ell)\right)^{\gamma}\right]^{1 / \gamma} \int_{\ell}^{u} \frac{1}{b^{1 / \gamma}(s)} d s .
$$

Letting $u \rightarrow \infty$, we see that

$$
-y^{(n-2)}(\ell) \leq\left[b(\ell)\left(y^{(n-1)}(\ell)\right)^{\gamma}\right]^{1 / \gamma} R(\ell) .
$$

From Lemma 1.2 we get

$$
y(\ell) \geq \frac{\lambda}{(n-2) !} \ell^{n-2} y^{(n-2)}(\ell)
$$

for all $\lambda \in(0,1)$ and every sufficiently large $\ell$. Next, we define

$$
\varphi(\ell)=\frac{b(\ell)\left(y^{(n-1)}(\ell)\right)^{\gamma}}{\left(y^{(n-2)}(\ell)\right)^{\gamma}}
$$

We note that $\varphi(\ell)<0$ for $\ell \geq \ell_{1}$ and

$$
\varphi^{\prime}(\ell)=\frac{\left(b(\ell)\left(y^{(n-1)}(\ell)\right)^{\gamma}\right)^{\prime}}{\left(y^{(n-2)}(\ell)\right)^{\gamma}}-\gamma \frac{b(\ell)\left(y^{(n-1)}(\ell)\right)^{\gamma+1}}{\left(y^{(n-2)}(\ell)\right)^{\gamma+1}} .
$$

From (1.1) and (2.12) we obtain

$$
\varphi^{\prime}(\ell) \leq-Q(\ell) \frac{y^{\gamma}(g(\ell, c))}{\left(y^{(n-2)} g(\ell, c)\right)^{\gamma}} \frac{\left(y^{(n-2)} g(\ell, c)\right)^{\gamma}}{\left(y^{(n-2)}(\ell)\right)^{\gamma}}-\gamma \frac{1}{b^{1 / \gamma}(\ell)} \varphi^{\frac{\gamma+1}{\gamma}}(\ell) .
$$


Hence (2.13) yields

$$
\varphi^{\prime}(\ell) \leq-Q(s)\left(\frac{\lambda_{2}}{(n-2) !} g^{n-2}(s, c)\right)^{\gamma}-\gamma \frac{1}{b^{1 / \gamma}(\ell)} \varphi^{\frac{\gamma+1}{\gamma}}(\ell) .
$$

Multiplying (2.14) by $R^{\gamma}(\ell)$ and integrating from $\ell_{2}$ to $\ell$, we obtain

$$
\begin{aligned}
& R^{\gamma}(\ell) \varphi(\ell)-R^{\gamma}\left(\ell_{2}\right) \varphi\left(\ell_{2}\right)+\gamma \int_{\ell_{2}}^{\ell} \frac{R^{\gamma-1}(s)}{b^{1 / \gamma}(s)} \varphi(s) d s \\
& \quad \leq-\int_{\ell_{2}}^{\ell} Q(s)\left(\frac{\lambda_{2}}{(n-2) !} g^{n-2}(s, c)\right)^{\gamma} R^{\gamma}(s) d s-\gamma \int_{\ell_{2}}^{\ell} \frac{\varphi^{\frac{1+\gamma}{\gamma}}(s)}{b^{1 / \gamma}(s)} R^{\gamma}(s) d s .
\end{aligned}
$$

Set

$$
U:=\frac{R^{\gamma-1}(s)}{b^{1 / \gamma}(s)}, \quad V:=\frac{R^{\gamma}(s)}{b^{1 / \gamma}(s)}, \quad y:=-\varphi(s) .
$$

By Lemma (1.3) we find

$$
\begin{aligned}
& \int_{\ell_{2}}^{\ell}\left[Q(s)\left(\frac{\lambda_{2}}{(n-2) !} g^{n-2}(s, c)\right)^{\gamma} R^{\gamma}(s)-\left(\frac{\gamma}{\gamma+1}\right)^{\gamma+1} \frac{b^{-1 / \gamma}(s)}{R(s)}\right] d s \\
& \quad \leq 1+R^{\gamma}\left(\ell_{2}\right) \varphi\left(\ell_{2}\right)
\end{aligned}
$$

for some constant $\lambda_{2} \in(0,1)$, which contradicts (2.2).

Theorem (2.1) is proved.

It is well known (see [3]) that the differential equation

$$
\left[a(\ell)\left(y^{\prime}(\ell)\right)^{\alpha}\right]^{\prime}+q(\ell) y^{\alpha}(\tau(\ell))=0, \quad \ell \geq \ell_{0}
$$

where $\alpha>0$ is the ratio of odd positive integers and $a, q \in C\left[\ell_{0}, \infty\right)$, is nonoscillatory if and only if there exist a number $\ell \geq \ell_{0}$ and a function $v \in C^{1}[\ell, \infty)$ satisfying the inequality

$$
v^{\prime}(\ell)+\alpha a^{\frac{-1}{\alpha}}(\ell)(v(\ell))^{\frac{(1+\alpha)}{\alpha}}+q(\ell) \leq 0 \quad \text { for }[\ell, \infty)
$$

In what follows, we compare the oscillatory behavior of (1.1) with the second-order halflinear equations of type (2.15).

Theorem 2.2 Let $\left(A_{1}\right),\left(A_{2}\right)$, and (1.2) hold. Assume that the differential equations

$$
\left[\frac{b(\ell)}{\ell^{2 \gamma}}\left(y^{\prime}(\ell)\right)^{\gamma}\right]^{\prime}+Q(\ell)\left(\frac{\lambda_{1} g^{3}(\ell, c)}{2 \ell^{3}}\right)^{\gamma} y^{\gamma}(\ell)=0
$$

and

$$
\left[b(\ell)\left(y^{\prime}(\ell)\right)^{\gamma}\right]^{\prime}+Q(\ell)\left(\frac{\lambda}{(n-2) !} g^{n-2}(\ell, c)\right)^{\gamma} y^{\gamma}(\ell)=0
$$

are oscillatory for some constants $\lambda, \lambda_{1} \in(0,1)$. Then every solution of $(1.1)$ is oscillatory. 
Proof We proceed as in the proof of Theorem (2.1). Setting $\delta(\ell)=1$ in (2.9), we get

$$
\omega^{\prime}(\ell)+\frac{1}{(n-4) !} \int_{\ell}^{\infty}(v-\ell)^{(n-4)} \sigma^{\frac{1}{\gamma}}(v) b(v)^{-1 / \gamma} d v \leq 0 .
$$

Thus, we see that equation (2.16) is nonoscillatory, a contradiction. From (2.14) we obtain

$$
\varphi^{\prime}(\ell)+Q(s)\left(\frac{\lambda_{1}}{(n-2) !} g^{n-2} g(s, c)\right)^{\gamma}+\gamma \frac{1}{b^{1 / \gamma}(\ell)} \varphi^{\frac{\gamma+1}{\gamma}}(\ell) \leq 0
$$

for every constant $\lambda_{1} \in(0,1)$. Thus we have that equation (2.17) is nonoscillatory for every constant $\lambda \in(0,1)$, a contradiction.

Theorem (2.2) is proved.

\section{Examples}

In this section, we give some examples to illustrate our main results.

Example 3.1 Consider the fourth-order differential equation

$$
\left(\mathbf{e}^{3 \ell}\left(y^{\prime \prime \prime}(\ell)\right)^{3}\right)^{\prime}+\int_{0}^{1} \frac{\ell \mathbf{e}^{\ell(\xi+3)}}{\mathbf{e}^{\ell}-1} y^{3}(\ell-\xi) d \xi=0, \quad \ell \geq 1 .
$$

Note that $\gamma=3, n=4, b(\ell)=\mathbf{e}^{3 \ell}, q(\ell, \xi)=\ell \mathbf{e}^{\ell(\xi+3)} /\left(\mathbf{e}^{\ell}-1\right), c=0, d=1, g(\ell, c)=\ell, R(\ell)=\mathbf{e}^{-\ell}$, and $Q(\ell)=\mathbf{e}^{3 \ell}$. If we choose $\delta(\ell)=1$, then it easy to see that conditions (2.1) and (2.2) hold. Hence, by Theorem 2.1, every solution of equation (3.1) is oscillatory.

Example 3.2 Consider the differential equation

$$
\left(\ell^{3}\left(y^{\prime \prime \prime}(\ell)\right)\right)^{\prime}+\int_{0}^{1} \frac{\nu \xi}{\ell} y\left(\frac{\ell-\xi}{2}\right) d \xi=0, \quad \ell \geq 1
$$

where $v>0$ is a constant. Note that $\gamma=1, n=4, b(\ell)=\ell^{3}, q(\ell, \xi)=\xi \nu / \ell, c=0, d=1$, $g(\ell, c)=\ell / 2, Q(\ell)=v / 2 \ell$, and $R(\ell)=1 / 2 s^{2}$. If we set $\delta(\ell)=1$, then we have

$$
\begin{aligned}
& \int_{\ell_{0}}^{\infty}\left[Q(s)\left(\frac{\lambda_{2}}{(n-2) !} g^{n-2}(s, c)\right)^{\gamma} R^{\gamma}(s)-\left(\frac{\gamma}{\gamma+1}\right)^{\gamma+1} \frac{b^{-1 / \gamma}(s)}{R(s)}\right] d s \\
& \quad=\left(\frac{\nu \lambda_{2}}{32}-\frac{1}{2}\right) \int_{\ell_{0}}^{\infty} \frac{1}{\ell} d \ell \\
& \quad=\infty
\end{aligned}
$$

for $v>\frac{16}{\lambda_{2}}$ and some constant $\lambda_{2} \in(0,1)$. Hence, by Theorem 2.1, every solution of equation (3.2) is oscillatory if $v>\frac{16}{\lambda_{2}}$.

Remark 3.1 The results of [4] cannot confirm the conclusion in Example 3.2.

Remark 3.2 Our results supplement and improve the results obtained in [25]. 


\section{Conclusion}

In this paper, by employing a refinement of the generalized Riccatti transformations and new comparison principles we have established new oscillation criteria for higher-order nonlinear differential equations with distributed delay. Further, we can consider the case of $g(\ell, \xi) \geq \ell$, and we can try to get some oscillation criteria of equation (1.1) in the future work.

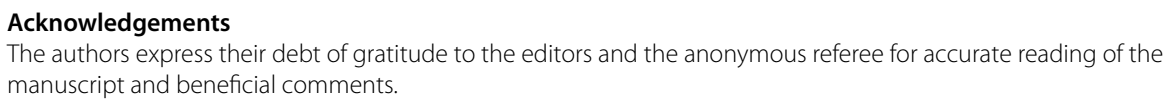

\section{Funding}

The authors received no direct funding for this work.

\section{Competing interests}

The authors declare that there are no competing interests between them.

\section{Authors' contributions}

The authors declare that they read and approved the final manuscript.

\section{Author details}

'Department of Mathematics, Faculty of Education, Hadhramout University, Hadhramout, Yemen. ${ }^{2}$ Department of Mathematics, Faculty of Science, Mansoura University, Mansoura, Egypt.

\section{Publisher's Note}

Springer Nature remains neutral with regard to jurisdictional claims in published maps and institutional affiliations.

Received: 8 August 2018 Accepted: 18 February 2019 Published online: 04 March 2019

\section{References}

1. Agarwal, R., Grace, S., O'Regan, D.: Oscillation Theory for Difference and Functional Differential Equations. Kluwer Academic, Dordrecht (2000)

2. Agarwal, R.P., Bohner, M., Li, T.: Oscillatory behavior of second-order half-linear damped dynamic equations. Appl. Math. Comput. 254, 408-418 (2015)

3. Agarwal, R.P., Zhang, C., Li, T.: Some remarks on oscillation of second order neutral differential equations. Appl. Math. Comput. 274, 178-181 (2016)

4. Bazighifan, O.: Oscillatory behavior of higher-order delay differential equations. Gen. Lett. Math. 2, 105-110 (2017)

5. Elabbasy, E.M., Moaaz, O., Bazighifan, O.: Oscillation solution for higher-order delay differential equations. J. King Abdulaziz Univ. 29, 45-52 (2017)

6. Elabbasy, E.M., Moaaz, O., Bazighifan, O.: Oscillation of fourth-order advanced differential equations. J. Mod. Sci. Eng. 3, 64-71 (2017)

7. Elabbasy, E.M., Moaaz, O., Bazighifan, O.: Oscillation criteria for fourth-order nonlinear differential equations. Int. J. Mod. Math. Sci. 15, 50-57 (2017)

8. Grace, S., Agarwal, R., Graef, J.: Oscillation theorems for fourth order functional differential equations. J. Appl. Math. Comput. 30, 75-88 (2009)

9. Gyori, I., Ladas, G.: Oscillation Theory of Delay Differential Equations with Applications. Clarendon Press, Oxford (1991)

10. Kiguradze, I., Chanturia, T.: Asymptotic Properties of Solutions of Nonautonomous Ordinary Differential Equations. Kluwer Academic, Drodrcht (1993)

11. Ladde, G., Lakshmikantham, V., Zhang, B.: Oscillation Theory of Differential Equations with Deviating Arguments. Marcel Dekker, NewYork (1987)

12. Li, T., Baculikova, B., Dzurina, J., Zhang, C.: Oscillation of fourth order neutral differential equations with $p$-Laplacian like operators. Bound. Value Probl. 2014, 56 (2014)

13. Li, T., Rogovchenko, Y.V.: Oscillation of second-order neutral differential equations. Math. Nachr. 288, 1150-1162 (2015)

14. Li, T., Rogovchenko, Y.V.: Oscillation criteria for even-order neutral differential equations. Appl. Math. Lett. 61, 35-41 (2016)

15. Li, T., Rogovchenko, Y.V.: On asymptotic behavior of solutions to higher-order sublinear Emden-Fowler delay differential equations. Appl. Math. Lett. 67, 53-59 (2017)

16. Li, T., Rogovchenko, Y.V.: Oscillation criteria for second-order superlinear Emden-Fowler neutral differential equations. Monatshefte Math. 184, 489-500 (2017)

17. Li, T., Rogovchenko, Y.V., Zhang, C.: Oscillation results for second-order nonlinear neutral differential equations. Adv. Differ. Equ. 2013, 336 (2013)

18. Li, T., Zhang, C., Thandapani, E.: Asymptotic behavior of fourth-order neutral dynamic equations with noncanonical operators. Taiwan. J. Math. 18, 1003-1019 (2014)

19. Moaaz, O., Elabbasy, E.M., Bazighifan, O.: On the asymptotic behavior of fourth-order functional differential equations. Adv. Differ. Equ. 2017, 261 (2017) 
20. Philos, C.: On the existence of nonoscillatory solutions tending to zero at $\infty$ for differential equations with positive delay. Arch. Math. (Basel) 36, 168-178 (1981)

21. Tunc, C., Bazighifan, O.: Some new oscillation criteria for fourth-order neutral differential equations with distributed delay. Electron. J. Math. Anal. Appl. 7, 235-241 (2019)

22. Zhang, C., Agarwal, R., Bohner, M., Li, T.: New results for oscillatory behavior of even-order half-linear delay differential equations. Appl. Math. Lett. 26, 179-183 (2013)

23. Zhang, C., Agarwal, R.P., Bohner, M., Li, T.: Oscillation of second-order nonlinear neutral dynamic equations with noncanonical operators. Bull. Malays. Math. Sci. Soc. (2) 38, 761-778 (2015)

24. Zhang, C., Li, T., Saker, S.: Oscillation of fourth-order delay differential equations. J. Math. Sci. 201, 296-308 (2014)

25. Zhang, C., Li, T., Sun, B., Thandapani, E.: On the oscillation of higher-order half-linear delay differential equations. Appl. Math. Lett. 24, 1618-1621 (2011)

\section{Submit your manuscript to a SpringerOpen ${ }^{\circ}$} journal and benefit from:

- Convenient online submission

- Rigorous peer review

Open access: articles freely available online

- High visibility within the field

Retaining the copyright to your article

Submit your next manuscript at $\gg$ springeropen.com 